\title{
Bulk viscosity of strange matter and r-modes in neutron stars
}

\section{Debarati Chatterjee}

Theory Division and Centre for Astroparticle Physics Saha Institute of Nuclear Physics, Kolkata-700064, India

E-mail: debarati.chatterjee@saha.ac.in

\section{Debades Bandyopadhyay*}

Theory Division and Centre for Astroparticle Physics Saha Institute of Nuclear Physics, Kolkata-700064, India

E-mail: debades.bandyopadhyayesaha.ac.in

\begin{abstract}
We discuss bulk viscosity due to non-leptonic processes involving hyperons and Bose-Einstein condensate of negatively charged kaons in neutron stars. It is noted that the hyperon bulk viscosity coefficient is a few order of magnitude larger than that of the case with the condensate. Further it is found that the hyperon bulk viscosity is suppressed in a superconducting phase. The hyperon bulk viscosity efficiently damps the r-mode instability in neutron stars irrespective of whether a superconducting phase is present or not in neutron star interior.
\end{abstract}

10th Symposium on Nuclei in the Cosmos

July 27 - August 1, 2008

Mackinac Island, Michigan, USA

${ }^{*}$ Speaker. 


\section{Introduction}

Neutron stars pulsate in various modes due to its fluid perturbation. Those oscillatory modes are classified according to different restoring forces. The study of Coriolis restored r-mode in neutron stars has generated tremendous interest in recent times [1]. The mode becomes unstable due to gravitational radiation. R-modes of rotating neutron stars are important sources of detectable gravitational waves. The $l=m=2 \mathrm{r}$-mode frequency $\left(\omega_{r}\right)$ is related to the angular frequency of the compact star as $\omega_{r}=\frac{2 m}{l(l+1)} \Omega$ [1]. The calculation of $\Omega$ depends on the equation of state (EoS) as it will be shown in Figure 3. Therefore, r-modes, if detected, would shed light on the EoS and composition of matter in neutron star interior.

Depending on the nature of strong interaction, different novel phases with large strangeness fraction such as, hyperon matter, Bose-Einstein condensate of negatively charged kaons and quark phase may appear in neutron star interior. The r-mode instability could be effectively suppressed by bulk viscosity due to non-leptonic processes including hyperons [2, 3] in neutron star interior. Here we discuss bulk viscosity coefficients and the corresponding damping time scales due to nonleptonic weak processes involving hyperons and negatively charged kaons. Next we demonstrate the effect of bulk viscosity on the damping of the gravitational radiation driven r-mode instability in neutron stars. Further we discuss the influence of antikaon condensate on the hyperon bulk viscosity.

\section{Composition and EoS}

The knowledge about composition and EoS of dense matter is important to investigate the oscillatory modes of compact stars. Here we consider three different compositions for $\beta$-equilibrated and charge neutral neutron star matter (i) including $n, p, \Lambda$ hyperons, electrons and muons, (ii) undergoing a first order phase transition from nuclear matter to $K^{-}$condensed matter and (iii) involving a first order phase transition from $\Lambda$ hyperon matter to $K^{-}$condensed matter. The baryon-baryon interaction is mediated by the exchange of $\sigma, \omega$ and $\rho$ mesons and two strange mesons, scalar meson, $f_{0}(975)$ and the vector meson $\phi(1020)[4,5]$. Similarly (anti)kaon-baryon interaction is treated in the same footing $[6,7,8,9,10]$ as baryon-baryon interaction. Nucleonmeson coupling constants are determined by reproducing nuclear matter saturation properties such as binding energy $E / B=-16.3 \mathrm{MeV}$, baryon density $n_{0}=0.153 \mathrm{fm}^{-3}$, asymmetry energy coefficient $a_{\text {asy }}=32.5 \mathrm{MeV}$, and effective nucleon masses $m_{N}^{*} / m_{N}=0.78,0.70$ corresponding two values of incompressibility of nuclear matter $K=240,300 \mathrm{MeV}$. Parameters of the model are tabulated in Ref.[11]. Further kaon-meson and hyperon-meson coupling constants are obtained from Ref. $[4,10,12]$. In this calculation, we take the value of $\Lambda$ hyperon potential depth of $-30 \mathrm{MeV}$ in nuclear matter and the value of antikaon optical potential depth at normal nuclear matter density $U_{\bar{K}}\left(n_{0}\right)=-120,-160 \mathrm{MeV}$.

For $K=240,300 \mathrm{MeV}, \Lambda$ hyperons appear at $2.6 n_{0}$ and $2.3 n_{0}$, respectively whereas $K^{-}$condensation sets in at $3.26 n_{0}$ for $K=240 \mathrm{MeV}$ and $U_{\bar{K}}\left(n_{0}\right)=-120 \mathrm{MeV}$ and at $2.23 n_{0}$ for $K=300$ $\mathrm{MeV}$ and $U_{\bar{K}}\left(n_{0}\right)=-160 \mathrm{MeV}$. 


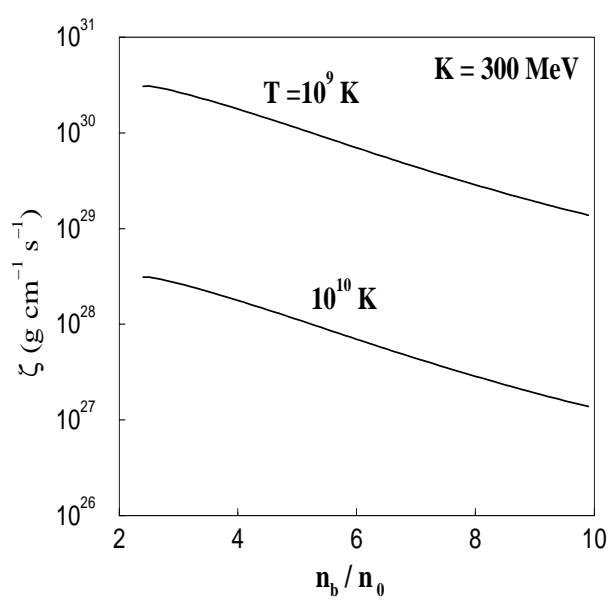

Figure 1: Hyperon bulk viscosity coefficient is plotted with normalised baryon density for different temperatures.

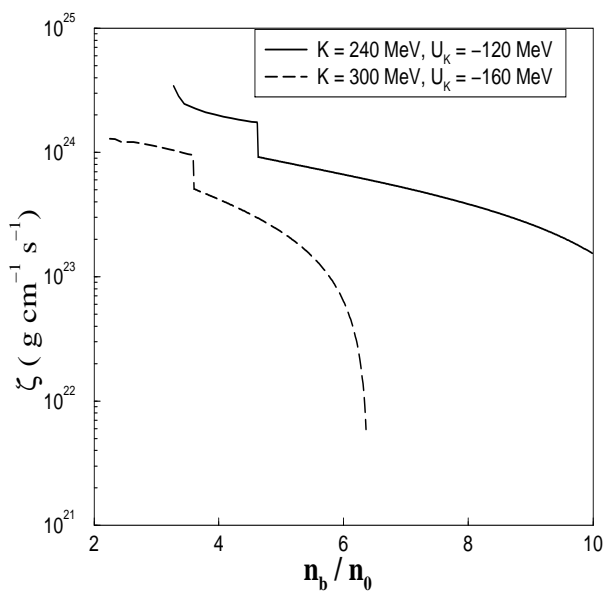

Figure 2: Antikaon bulk viscosity coefficient is shown as a function of normalised baryon density for different values of $K$ and $U_{\bar{K}}\left(n_{0}\right)$.

\section{Bulk Viscosity and Critical Angular Velocity}

Energy dissipation connected with pressure and density variations due to the r-mode results in bulk viscosity. The system goes out of chemical equilibrium due to those variations. Weak processes bring it back to the equilibrium. We calculate bulk viscosity due to the following two weak processes,

$$
\begin{gathered}
n+p \rightleftharpoons p+\Lambda, \\
n \rightleftharpoons p+K^{-} .
\end{gathered}
$$

The real part of the bulk viscosity coefficient is given by $[13,14]$

$$
\zeta=\frac{P\left(\gamma_{\infty}-\gamma_{0}\right) \tau}{1+(\omega \tau)^{2}}
$$

The relaxation times $(\tau)$ for the above mentioned weak processes are taken from Ref.[12, 15, 16]. Bulk viscosity coefficients due to weak processes including $\Lambda$ hyperons and the condensate as a 
function of normalised baryon density are displayed in Figure 1 and Figure 2 respectively. Hyperon bulk viscosity in Fig. 1 increases with decreasing temperature whereas the antikaon bulk viscosity does not vary with temperature because the condensate is treated at zero temperature [12]. Further it is observed that the antikaon bulk viscosity coefficient is several orders of magnitude lower than the hyperon bulk viscosity $[12,15,16]$.

The critical angular velocity of a neutron star is calculated by solving the overall $r$-mode timescale $\left(\tau_{r}\right)[12,15,16]$,

$$
\frac{1}{\tau_{r}}=-\frac{1}{\tau_{G R}}+\frac{1}{\tau_{B}}+\frac{1}{\tau_{U}}=0 .
$$

Neutron stars which are rotating above critical angular velocity $\left(\Omega_{C}\right)$, are unstable due to grav-

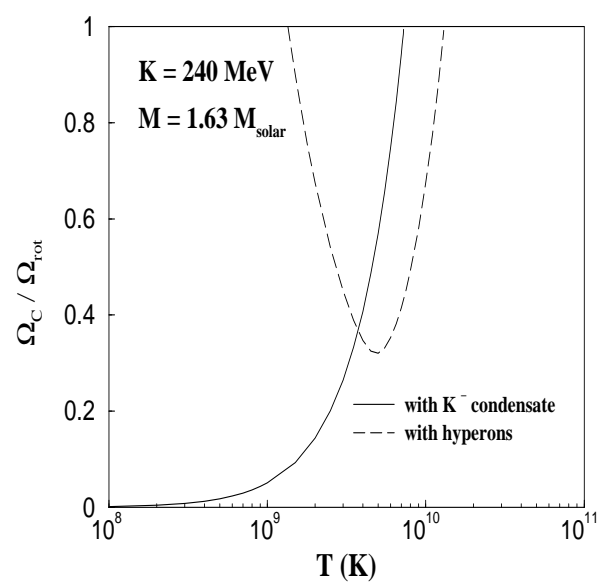

Figure 3: Critical angular velocity of $1.63 M_{\odot}$ neutron star is shown as a function of temperature.

itational radiation whereas they are stable below $\Omega_{C}$. Critical angular velocities as a function of temperature for cases (i) and (ii) are exhibited in Figure 3. For case (i) denoted by the dashed curve, the r-mode instability of the neutron star having mass $1.63 M_{\odot}$ is damped in the temperature regime above $10^{10} \mathrm{~K}$ because the damping time scale $\left(\tau_{U}\right)$ due to the modified Urca process [15] involving nucleons is comparable with that of the gravitational growth time scale $\left(\tau_{G R}\right)$. The mode is damped by the hyperon bulk viscosity below $10^{10} \mathrm{~K}$. On the other hand, the antikaon bulk viscosity can not damp the r-mode as it is shown by the solid curve in Fig. 3.

Next we focus on the hyperon bulk viscosity due to the non-leptonic process in Eq. (3.1) in the presence of a $K^{-}$condensate. Figure 4 shows the hyperon bulk viscosity coefficient as a function of normalised baryon density at a temperature $T=2 \times 10^{9} \mathrm{~K}$. The bulk viscosity in the hadronic (bold solid line) and $\mathrm{K}^{-}$condensed (light solid line) parts of the mixed phase and the pure condensed phase (dashed line) are shown in the figure. The hyperon bulk viscosity in the antikaon condensed matter is suppressed than that of the hadronic phase. It is observed that the hyperon bulk viscosity in $K^{-}$condensed matter might damp the r-mode instability effectively [17].

\section{Summary}

We have investigated bulk viscosity due to non-leptonic processes involving $\Lambda$ hyperons and $K^{-}$condensate in neutron stars. It is noted that the hyperon bulk viscosity coefficient is a few 




Figure 4: Hyperon bulk viscosity coefficient in $K^{-}$condensed matter is shown as a function of normalised baryon density.

orders of magnitude higher than the antikaon bulk viscosity coefficient. Hyperon bulk viscosity efficiently damps the r-mode instability.

\section{References}

[1] Andersson N and Kokkotas K D 2001 Int. J. Mod. Phys. D 10381

[2] Jones P B 2001 Phys. Rev. Lett. 861384

[3] Jones P B 2001 Phys. Rev. D 64084003

[4] Schaffner J and Mishustin I N 1996 Phys. Rev. C 531416

[5] Boguta J and Bodmer A R 1977 Nucl. Phys. A 292413

[6] Glendenning N K and Schaffner-Bielich J 1998 Phys. Rev. Lett. 814564

[7] Glendenning N K and Schaffner-Bielich J 1999 Phys. Rev. C 60025803

[8] Pal S, Bandyopadhyay D and Greiner W 2000 Nucl. Phys. A 674553

[9] Banik S and Bandyopadhyay D 2001 Phys. Rev. C 63035802

[10] Banik S and Bandyopadhyay D 2001 Phys. Rev. C 64055805

[11] Glendenning N K and Moszkowski S A 1991 Phys. Rev. Lett. 672414

[12] Chatterjee D and Bandyopadhyay D 2007 Phys. Rev. D 75123006

[13] Lindblom L and Owen B J 2002 Phys. Rev. D 65063006

[14] Nayyar M and Owen B J 2006 Phys. Rev. D 73084001

[15] Chatterjee D and Bandyopadhyay D 2006 Phys. Rev. D 74023003

[16] Chatterjee D and Bandyopadhyay D 2007 Astrophys. Space Sc. 308451

[17] Chatterjee D and Bandyopadhyay D 2008 ApJ 680686 